\title{
Vibrational Spectroscopic Investigation and Conformational Analysis of Methacrylamidoantipyrine: A Comparative Density Functional Study
}

\author{
Özgür Alver, ${ }^{1,2}$ Mehmet Fatih Kaya, ${ }^{3}$ Metin Bilge, ${ }^{4}$ and Cemal Parlak ${ }^{3}$ \\ ${ }^{1}$ Department of Physics, Science Faculty, Anadolu University, 26470 Eskişehir, Turkey \\ ${ }^{2}$ Plant, Drug and Scientific Research Centre, Anadolu University, 26470 Eskişehir, Turkey \\ ${ }^{3}$ Department of Physics, Dumlupinar University, 43100 Kütahya, Turkey \\ ${ }^{4}$ Department of Physics, Sciences Faculty, Ege University, 35100 Izmir, Turkey
}

Correspondence should be addressed to Mehmet Fatih Kaya; mehmetfatihkaya88@gmail.com

Received 9 April 2013; Accepted 23 June 2013

Academic Editors: M. Koyama and A. M. Lamsabhi

Copyright (C) 2013 Özgür Alver et al. This is an open access article distributed under the Creative Commons Attribution License, which permits unrestricted use, distribution, and reproduction in any medium, provided the original work is properly cited.

\begin{abstract}
FT-IR and Raman spectra of methacrylamidoantipyrine (MAAP) have been reported in the region of 4000-10 $\mathrm{cm}^{-1}$ and $4000-100$ $\mathrm{cm}^{-1}$, respectively. The optimized geometric parameters, conformational analysis, normal mode frequencies, and corresponding vibrational assignments of MAAP $\left(\mathrm{C}_{15} \mathrm{H}_{17} \mathrm{~N}_{3} \mathrm{O}_{2}\right)$ have been examined by means of density functional theory (DFT) method using the Becke-3-Lee-Yang-Parr (B3LYP) exchange-correlation functional and the $6-31 \mathrm{G}^{++}(\mathrm{d}, \mathrm{p})$ basis sets. Vibrational assignments have been made on the basis of potential energy distribution (PED) and the thermodynamics functions, and the highest occupied and the lowest unoccupied molecular orbitals (HOMO and LUMO) of MAAP have been predicted. Calculations are carried out with the possible seven (amide-1-5 and imide-1-2) conformational isomers of MAAP. Comparison between the experimental and theoretical results indicates that the B3LYP method provides satisfactory evidence for the prediction of vibrational wavenumbers, and the amide- 1 conformational isomer is supposed to be the most stable form of MAAP.
\end{abstract}

\section{Introduction}

The MAAP, which has a $\pi$ electron rich aromatic ring, was first synthesized using antipyrine, a hydroxy radical capture and spectroscopic reagent for phenols, and used for a method described for removal of phenolic compounds with nitrophenol imprinted polymer based on $\pi-\pi$ and hydrogen bonding interactions [1]. Then, it was used in analyzing the selective binding behavior of paraoxon and parathion compounds on surface imprinted polymers prepared using both charge transfer (MAAP) and ligand-exchange (MAAPGd) monomers [2]. Today, one can easily reach many papers published where it is one of the most important organic chemical product for the molecularly imprinted polymers [1-5]. For instance, it has been used in researching the biomimetic immunoassay based on molecularly imprinted polymer [3], the 8-Hydroxy-2'-deoxyguanosine (8-OHdG), which is one of the most abundant oxidative DNA lesions resulting from reactive oxygen species, and the 8-OHdG level in blood serum from a breast cancer patient [4], and new 8-OHdG imprinted quartz crystal microbalance sensor [5]. Furthermore, it is taken part in adsorption of phenol and its derivatives from water using synthetic resins and low-cost natural adsorbents [6].

Vibrational spectroscopy has been widely used as the standard tool for structural characterization of molecular systems together with DFT calculations [7-14]. DFT is popular as it is a cost-effective procedure for studying the physical properties of molecules. Unlike the Hartree Fock theory, DFT recovers electron correlation in the self-consistent KohnSham procedure through the functions of electron density, so it is a cost-effective and reliable method. The DFT/B3LYP model exhibits good performance particularly on vibrational frequencies and geometries of organic compounds [7-14].

Though MAAP has wide applications in science, to the best of our knowledge, there is limited information 
available in the literature about its spectroscopic properties. A detailed, quantum chemical study will be useful in making assignments to the fundamental normal modes and in explaining the obtained experimental data of MAAP. Furthermore, theoretically and experimentally presented data may be helpful in the context of the further studies of MAAP. For the above goals, we have reported vibrational spectra of MAAP. Vibrational frequencies with PED values, HOMO and LUMO data, structural parameters, and some thermodynamics functions of MAAP are also calculated for the most stable conformational isomer of MAAP by means of $\mathrm{B} 3 \mathrm{LYP} / 6-31 \mathrm{G}^{++}(\mathrm{d}, \mathrm{p})$ level. The results of the theoretical and spectroscopic studies are reported here.

\section{Experimental}

MAAP was prepared according to the published procedure [1] and used without further purification. FT-MIR and FIR spectra were recorded in the region of $4000-400 \mathrm{~cm}^{-1}$ and $400-10 \mathrm{~cm}^{-1}$ with Bruker Optics IFS66v/s FTIR spectrometer at a resolution of $2 \mathrm{~cm}^{-1}$. Raman spectrum was obtained using a Bruker Senterra Dispersive Raman microscope spectrometer with $785 \mathrm{~nm}$ excitation from a 3B diode laser having $3 \mathrm{~cm}^{-1}$ resolution in the spectral region of $4000-100 \mathrm{~cm}^{-1}$.

\section{Calculations}

All the calculations were performed using Gaussian 09.A1 program [15] on HP DL380G7 server system, and GaussView 5.0.8 [16] was used for visualization of the structure and simulated vibrational spectra. Many possible conformational isomers could be proposed for MAAP; however, the five amide and two imide conformational isomers of MAAP (Figure 1) were chosen using HF/6-31G(d,p) level by Spartan 10 program [17]. For these calculations, the possible seven conformational isomers of MAAP were first optimized in the gas phase at B3LYP level of theory using $6-31 \mathrm{G}^{++}(\mathrm{d}, \mathrm{p})$ basis set, and no geometric restrictions were applied. Its amide1 conformational isomer was found more stable than the others (Figure 1). According to the theoretical calculations, the vibrational frequencies and assignments of the two conformational isomers amide- 1 and amide- 2 are about the same. So, it has been given the data for one of them.

Therefore, after the optimization, harmonic vibrational frequencies and corresponding vibrational intensities for the amide-1 conformational isomer of MAAP were calculated by using the same method and basis set and then scaled by 0.955 (above $1800 \mathrm{~cm}^{-1}$ ) and 0.977 (under $1800 \mathrm{~cm}^{-1}$ ) $[10,13]$. In order to show the relative contributions of the redundant internal coordinates to each normal vibrational mode of the molecule, PED calculations were carried out by the vibrational energy distribution analysis 4 (VEDA) [18].

\section{Results and Discussion}

4.1. Geometrical Structures. To clarify the vibrational frequencies, it is essential to examine the geometry of any compound, as small changes in geometry can potentially cause substantial differences in frequencies. Gibbs free energy and relative stability of the optimized geometries in gas phase for seven conformational isomers of MAAP with B3LYP/6$31 \mathrm{G}^{++}(\mathrm{d}, \mathrm{p})$ method are given in Figure 1. Regarding the calculated free energies, all conformational isomers relative to the amide- 1 and amide- 2 forms of MAAP could be neglected for the calculation of equilibrium constant since their energy differences are larger than $2 \mathrm{kcal} / \mathrm{mol}$ [10-14]. The amide-1 conformational isomer of MAAP is more stable than amide2 by $0.03 \mathrm{kcal} / \mathrm{mol}$. Consequently, MAAP in the gas phase prefers the amide- 1 and amide- 2 conformational isomers with preference of $51 \%$ and $49 \%$, respectively.

Some of the optimized geometric parameters such as bond lengths and bond angles calculated by B3LYP/6$31 \mathrm{G}^{++}(\mathrm{d}, \mathrm{p})$ are listed in Table 1 for the most stable conformational isomer (amide-1) of MAAP. To the best of our knowledge, experimental data on the geometric structure of MAAP is not available in the literature. However, some geometric parameters for 4-([(1E)-(2-hydroxynaphthyl)methylidene]amino)-1,5-dimethyl-2-phenyl-2,3-dihyrdo-1H-pyrazol3-one and p-methacryloylaminophenylarsonic acid monomer were identified in previously reported studies $[19,20]$. Henceforth, the theoretical results have been compared with data for some parts of the related molecules as given in Table 1 .

Generally, it is expected that the bond distances calculated by electron correlated methods are longer than the experimental distance. This situation can be seen in Table 1 as expected. Overall, the calculated bond lengths are in good agreement with experimental results. The mean absolute deviation (MAD) and root mean square deviation (RMSD) of bond lengths are 0.022 and $0.032 \AA$, respectively. The biggest difference between the experimental and calculated bond distances is $0.061 \AA$. As can be seen in Table 1, big differences are observed in the calculated C6-N5-C7, C2-C1-N20 and C7-C1-N20 bond angles compared to experimental values. The calculated C2-C1-N20 and C6-N5-C7 bond angles are about $11^{\circ}$ smaller than the experimental result whereas the calculated C7-C1-N20 angle is about $11^{\circ}$ larger than the experimental value. All the other bond angles are reasonably close to the experimental data.

The MAD and RMSD of bond angles are 3.15 and $4.96^{\circ}$, respectively. The observed differences in bond distances and angles are not due to the theoretical shortcomings, as experimental results are also subject to variations owing to the insufficient data to calculate equilibrium structure which are sometimes averaged over zero point vibrational motion. Furthermore, it can be noted that theoretical results have been compared with available experimental data.

For the optimized geometric parameters, magnitude of dihedral angles, $\mathrm{D}(10 ; 11 ; 12 ; 13)=0.70^{\circ}, \mathrm{D}(1 ; 7 ; 4 ; 5)=$ $177.49^{\circ}, \mathrm{D}(8 ; 7 ; 5 ; 4)=178.46^{\circ}, \mathrm{D}(20 ; 1 ; 2 ; 4)=178.68^{\circ}, \mathrm{D}$ $(1 ; 20 ; 15 ; 16)=165.90^{\circ}, \mathrm{D}(17 ; 16 ; 15 ; 18)=175.60^{\circ}, \mathrm{D}(14$; $9 ; 5 ; 4)=21.60^{\circ}, \mathrm{D}(1 ; 2 ; 3 ; 7)=173.40^{\circ}, \mathrm{D}(15 ; 16 ; 18 ; 20)=$ $17.85^{\circ}, \mathrm{D}(1 ; 20 ; 15 ; 19)=15.35^{\circ}$, and $\mathrm{D}(15 ; 16 ; 17 ; 19)=13.24^{\circ}$, indicate that a large part of molecule is nearly in the same plane. In this compound, two intramolecular hydrogen bonds

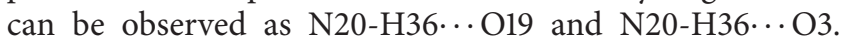
Theoretical results are N20-H36: $3.17 \AA / \mathrm{N} 20-\mathrm{H} 36-\mathrm{O} 19: 25.1^{\circ}$ 
TABLE 1: Some optimized geometric parameters of MAAP.

\begin{tabular}{|c|c|c|c|}
\hline \multirow{2}{*}{ Parameters } & \multicolumn{3}{|c|}{ B3LYP/6-31G ${ }^{++}(\mathrm{d}, \mathrm{p})$} \\
\hline & Amide-1 & Ref. [19] & Ref. [20] \\
\hline \multicolumn{4}{|l|}{ Bond lengths $(\AA)$} \\
\hline $\mathrm{C} 1-\mathrm{C} 2$ & 1.457 & 1.426 & \\
\hline $\mathrm{C} 1-\mathrm{C} 7$ & 1.362 & 1.369 & \\
\hline $\mathrm{C} 1-\mathrm{N} 20$ & 1.400 & 1.394 & 1.407 \\
\hline $\mathrm{C} 2-\mathrm{O} 3$ & 1.232 & 1.239 & \\
\hline $\mathrm{C} 2-\mathrm{N} 4$ & 1.400 & 1.395 & \\
\hline N4-N5 & 1.414 & 1.399 & \\
\hline N4-C9 & 1.420 & 1.422 & \\
\hline N5-C6 & 1.474 & 1.452 & \\
\hline N5-C7 & 1.406 & 1.362 & \\
\hline $\mathrm{C} 7-\mathrm{C} 8$ & 1.494 & 1.487 & \\
\hline $\mathrm{C} 15-\mathrm{C} 16$ & 1.508 & & 1.482 \\
\hline $\mathrm{C} 15-\mathrm{O} 19$ & 1.229 & & 1.227 \\
\hline $\mathrm{C} 15-\mathrm{N} 20$ & 1.377 & & 1.359 \\
\hline $\mathrm{C} 16-\mathrm{C} 17$ & 1.506 & & 1.426 \\
\hline $\mathrm{C} 16-\mathrm{C} 18$ & 1.342 & & 1.403 \\
\hline \multicolumn{4}{|l|}{ Bond angles $\left({ }^{\circ}\right)$} \\
\hline $\mathrm{C} 2-\mathrm{C} 1-\mathrm{N} 20$ & 117.8 & 128.8 & \\
\hline $\mathrm{C} 7-\mathrm{C} 1-\mathrm{N} 20$ & 133.0 & 122.0 & \\
\hline $\mathrm{C} 1-\mathrm{C} 2-\mathrm{O} 3$ & 128.2 & 132.3 & \\
\hline $\mathrm{C} 1-\mathrm{C} 2-\mathrm{N} 4$ & 105.0 & 104.8 & \\
\hline $\mathrm{O} 3-\mathrm{C} 2-\mathrm{N} 4$ & 126.7 & 122.9 & \\
\hline $\mathrm{C} 2-\mathrm{N} 4-\mathrm{N} 5$ & 109.8 & 109.4 & \\
\hline $\mathrm{C} 2-\mathrm{N} 4-\mathrm{C} 9$ & 125.1 & 127.1 & \\
\hline N4-N5-C6 & 113.1 & 119.6 & \\
\hline N4-N5-C7 & 106.7 & 107.5 & \\
\hline C6-N5-C7 & 117.3 & 128.9 & \\
\hline C1-C7-N5 & 109.3 & 109.2 & \\
\hline N5-C7-C8 & 119.2 & 121.7 & \\
\hline C16-C15-O19 & 121.3 & & 122.1 \\
\hline $\mathrm{C} 16-\mathrm{C} 15-\mathrm{N} 20$ & 115.9 & & 115.6 \\
\hline O19-C15-N20 & 122.8 & & 122.3 \\
\hline C15-C16-C17 & 114.8 & & 116.3 \\
\hline C15-C16-C18 & 121.7 & & 120.7 \\
\hline C17-C16-C18 & 123.4 & & 123.0 \\
\hline $\mathrm{C} 1-\mathrm{N} 20-\mathrm{C} 15$ & 126.2 & 121.4 & 128.2 \\
\hline
\end{tabular}

and N20-H36: $2.52 \AA / \mathrm{N} 20-\mathrm{H} 36-\mathrm{O} 19: 100.6^{\circ}$. The complete set of optimized geometric parameters for the amide-1 conformational isomer of MAAP is given in Table 3. Several thermodynamic parameters (zero point energy, entropy, etc.) calculated by B3LYP/6-31G ${ }^{++}(\mathrm{d}, \mathrm{p})$ method are also presented in Table S1 (see Supplementary Material available online at http://dx.doi.org/10.1155/2013/386247). The total energy and change in total entropy for the amide- 1 conformational isomer of MAAP are at room temperature.

4.2. Vibrational Studies of MAAP. To the best of our knowledge, the vibrational wavenumbers and assignments of MAAP in the middle and far infrared regions of the spectrum have not been reported in the literature. But, a few selected bands of MAAP were reported by Ersöz et al. [1]. The measured and calculated vibrational frequencies for MAAP along with corresponding vibrational assignments and intensities are given in Table 2 .

The theoretical and experimental vibrational spectra of MAAP are shown in Figure 2. All calculated frequency values presented in this paper are obtained within the harmonic approximation. This allows us to identify vibrational motion in terms of independent vibrational modes, each of them is governed by a simple one-dimensional harmonic potential. It is difficult to determine the MAAP's vibrational assignments in the observed spectrum due to its low symmetry. Therefore, the assignments of vibrational modes for the amide1 conformational isomer of MAAP have been provided by VEDA 4 [18] in Table 2. The MAAP molecule consists of 37 atoms having 105 normal vibrational modes, and its most stable form belongs to the point group $\mathrm{C}_{1}$ with only identity (E) symmetry element or operation. According to the calculations, 18 normal vibrational modes of MAAP are below $400 \mathrm{~cm}^{-1}$ while 87 modes are between $4000 \mathrm{~cm}^{-1}$ and $400 \mathrm{~cm}^{-1}$.

The high wavenumber region contains characteristic wavenumbers of $\mathrm{NH}$ stretching that are observed at 3249 $\mathrm{cm}^{-1}$ (IR) and at $3254 \mathrm{~cm}^{-1}$ (R) as strong broad band. The $\mathrm{NH}$ infrared band is consistent with previously reported data for MAAP [1] where this band was found as $3260 \mathrm{~cm}^{-1}$. The corresponding scaled theoretical value for this mode is $3426 \mathrm{~cm}^{-1}$. The amide band is the most intense and is predominantly $\mathrm{C}=\mathrm{O}$ stretch supports also the amide form of MAAP. Hydrogen bonding plays an important role in the broadening of the spectral bands [21]. The broadening of the $\mathrm{NH}$ bands is attributed to the intramolecularly bonded hydrogen to the $\mathrm{C}=\mathrm{O}$ group. The $\mathrm{CH}$ stretching region encompasses four and six $\mathrm{CH}$ modes in IR and Raman spectrum, respectively. The vibrational bands at 3094, $3059 \mathrm{~cm}^{-1}$ in IR spectrum and 3080, 3044, and $3005 \mathrm{~cm}^{-1}$ in Raman spectrum are attributed to the $\mathrm{CH}$ stretching vibrations while the bands at $2969,2919 \mathrm{~cm}^{-1}$ in IR spectrum and 2968,2934 , and $2863 \mathrm{~cm}^{-1}$ in Raman spectrum are assigned to the $\mathrm{CH}_{2}$ and $\mathrm{CH}_{3}$ stretchings. Corresponding scaled calculated values for these bands are found as 3090, $3073,3062,3040,3012,2971,2914$, and $2892 \mathrm{~cm}^{-1}$. In the high wavenumber region of the spectra, the anharmonicity can explain substantial differences between the experimental and calculated values. Alternatively, these differences may be due to intra/intermolecular interactions or to the laser used for Raman.

Carbonyl stretchings are observed at $1673 \mathrm{~cm}^{-1}-\mathrm{IR}$ $\left(1683 \mathrm{~cm}^{-1}-\mathrm{R}\right)$ and at $1648 \mathrm{~cm}^{-1}-$ IR $\left(1631 \mathrm{~cm}^{-1}-\mathrm{R}\right)$ as intense bands in the vibrational spectra. The former band is clearly assigned to the carbonyl band at cyclic ketone position, whereas the latter is attributed to the amide carbonyl band. The corresponding scaled theoretical values of these modes are $1703 \mathrm{~cm}^{-1}$ and $1694 \mathrm{~cm}^{-1} . \mathrm{C}=\mathrm{C}$ stretchings are observed at $1620 \mathrm{~cm}^{-1}$ - IR and at $1592 \mathrm{~cm}^{-1}$ - IR (1605 and $\left.1570 \mathrm{~cm}^{-1}-\mathrm{R}\right)$ as a very strong band in the vibrational spectra. The former band is assigned to the methacrylate 
TABLE 2: Experimental and calculated vibrational wavenumbers $\left(\mathrm{cm}^{-1}\right)$ of MAAP.

\begin{tabular}{|c|c|c|c|c|c|c|c|}
\hline \multirow{2}{*}{ Mode } & \multirow{2}{*}{ P.E.D $(\geq 5 \%)$} & \multicolumn{2}{|c|}{ Experimental } & \multicolumn{4}{|c|}{$\mathrm{B} 3 \mathrm{LYP} / 6-31 \mathrm{G}^{++}(\mathrm{d}, \mathrm{p})$} \\
\hline & & IR & Raman & $\nu^{\alpha}$ & $\nu^{\beta}$ & $I_{\text {IR }}$ & $I_{\mathrm{R}}$ \\
\hline$v_{1}$ & $\nu \mathrm{NH}(100)$ & $3249 \mathrm{~s}, \mathrm{~b}$ & $3254 \mathrm{~s}, \mathrm{~b}$ & 3588 & 3426 & 38.29 & 16.36 \\
\hline$v_{2}$ & $\nu \mathrm{CH}(97)$ & & & 3239 & 3093 & 0.43 & 12.83 \\
\hline$v_{3}$ & $\nu \mathrm{CH}(100)$ & $3094 \mathrm{w}$ & & 3235 & 3090 & 13.53 & 17.90 \\
\hline$v_{4}$ & $\nu \mathrm{CH}(89)$ & & 3080 vs & 3218 & 3073 & 3.23 & 25.29 \\
\hline$\nu_{5}$ & $\nu \mathrm{CH}(89)$ & $3059 \mathrm{~m}$ & & 3207 & 3062 & 25.36 & 40.11 \\
\hline$v_{6}$ & $\nu \mathrm{CH}(90)$ & & & 3193 & 3049 & 13.87 & 25.64 \\
\hline$v_{7}$ & vCH(93) & & $3044 \mathrm{w}$ & 3183 & 3040 & 1.14 & 12.12 \\
\hline$v_{8}$ & vCH(87) & & & 3172 & 3029 & 2.42 & 13.11 \\
\hline$v_{9}$ & $\nu \mathrm{CH}(95)$ & & & 3167 & 3025 & 8.58 & 8.38 \\
\hline$v_{10}$ & vCH(99) & & $3005 \mathrm{~s}$ & 3154 & 3012 & 4.74 & 32.59 \\
\hline$v_{11}$ & $\nu \mathrm{CH}(94)$ & & & 3136 & 2995 & 14.79 & 17.50 \\
\hline$v_{12}$ & $\nu \mathrm{CH}(96)$ & & & 3132 & 2991 & 12.80 & 14.93 \\
\hline$v_{13}$ & $\nu \mathrm{CH}(99)$ & $2969 \mathrm{~s}$ & $2968 \mathrm{~m}$ & 3111 & 2971 & 12.02 & 17.64 \\
\hline$v_{14}$ & vCH(92) & & & 3104 & 2964 & 12.55 & 15.67 \\
\hline$v_{15}$ & $\nu \mathrm{CH}(91)$ & $2919 \mathrm{~s}$ & 2934 vs & 3051 & 2914 & 18.10 & 52.55 \\
\hline$v_{16}$ & $\nu \mathrm{CH}(98)$ & & & 3042 & 2905 & 21.81 & 39.66 \\
\hline$v_{17}$ & $\nu \mathrm{CH}(96)$ & & $2863 \mathrm{~m}$ & 3028 & 2892 & 50.02 & 29.76 \\
\hline$\nu_{18}$ & $\nu \mathrm{OC}(74)$ & 1673 vs & $1683 \mathrm{~s}$ & 1743 & 1703 & 152.72 & 26.56 \\
\hline$\nu_{19}$ & $\nu \mathrm{OC}(74)$ & 1648 vs & 1631 vs & 1734 & 1694 & 363.40 & 52.85 \\
\hline$v_{20}$ & $\nu \mathrm{CC}(69)-5$ & & & 1702 & 1663 & 40.11 & 111.41 \\
\hline$\nu_{21}$ & $\nu \mathrm{CC}(76)$ & 1620 vs & & 1691 & 1652 & 100.80 & 34.41 \\
\hline$v_{22}$ & $\nu \mathrm{CC}(65)+\delta \mathrm{HCC}(19)$ & 1592 vs & 1605 vs & 1647 & 1609 & 53.05 & 79.10 \\
\hline$v_{23}$ & $\nu \mathrm{CC}(69)+\delta \mathrm{HCC}(10)$ & & $1570 \mathrm{~m}$ & 1633 & 1595 & 2.13 & 4.99 \\
\hline$v_{24}$ & $\delta \mathrm{HNC}(58)+\nu \mathrm{CN}(10)$ & $1491 \mathrm{vs}$ & & 1556 & 1520 & 352.19 & 41.25 \\
\hline$v_{25}$ & $\delta \mathrm{HCC}(60)+\nu \mathrm{CC}(10)$ & & & 1531 & 1496 & 105.42 & 13.21 \\
\hline$v_{26}$ & $\delta \mathrm{HCH}(85)$ & & & 1515 & 1481 & 15.71 & 6.95 \\
\hline$v_{27}$ & $\delta \mathrm{HCH}(84)$ & & & 1500 & 1465 & 2.85 & 7.29 \\
\hline$v_{28}$ & $\delta \mathrm{HCC}(81)$ & 1455 vs & $1466 \mathrm{~m}, \mathrm{sh}$ & 1499 & 1465 & 68.70 & 2.59 \\
\hline$v_{29}$ & $\delta \mathrm{HCC}(60)+\nu \mathrm{CC}(23)$ & & & 1491 & 1457 & 7.98 & 1.88 \\
\hline$v_{30}$ & $\delta \mathrm{HCH}(78)$ & & & 1484 & 1450 & 4.60 & 13.15 \\
\hline$v_{31}$ & $\delta \mathrm{HCH}(87)$ & & & 1477 & 1443 & 12.74 & 7.51 \\
\hline$v_{32}$ & $\delta \mathrm{HCH}(77)$ & & $1446 \mathrm{w}$ & 1477 & 1443 & 4.71 & 5.30 \\
\hline$v_{33}$ & $\delta \mathrm{HCH}(80)$ & & & 1456 & 1423 & 8.18 & 7.40 \\
\hline$v_{34}$ & $\delta \mathrm{HCH}(75)$ & & $1426 \mathrm{~s}$ & 1454 & 1421 & 7.77 & 21.77 \\
\hline$v_{35}$ & $\delta \mathrm{HCH}(67)+\nu \mathrm{CC}(11)$ & & & 1433 & 1400 & 76.53 & 29.21 \\
\hline$v_{36}$ & $\delta \mathrm{HCH}(77)$ & & & 1414 & 1382 & 5.79 & 5.84 \\
\hline$v_{37}$ & $\nu \mathrm{CC}(30)+\delta \mathrm{HCH}(19)$ & $1407 \mathrm{~s}$ & & 1392 & 1360 & 178.18 & 19.77 \\
\hline$v_{38}$ & $\nu \mathrm{NC}(59)$ & $1368 \mathrm{~s}$ & 1368 vs & 1371 & 1339 & 88.20 & 122.68 \\
\hline$v_{39}$ & $\delta \mathrm{HCC}(63)+\nu \mathrm{CC}(22)$ & & & 1360 & 1329 & 1.59 & 2.24 \\
\hline$v_{40}$ & $\nu \mathrm{CC}(52)+\delta \mathrm{HCC}(21)$ & 1310 vs, sh & $1303 \mathrm{~s}$ & 1340 & 1309 & 78.89 & 32.98 \\
\hline$v_{41}$ & $\nu \mathrm{CC}(42)+\delta \mathrm{HCC}(18)$ & & & 1325 & 1295 & 7.14 & 10.34 \\
\hline$v_{42}$ & $\nu \mathrm{NC}(39)$ & $\begin{array}{l}1295 \text { vs } \\
1285 \text { vs }\end{array}$ & $1245 \mathrm{w}, \mathrm{sh}$ & 1292 & 1263 & 218.19 & 34.06 \\
\hline$v_{43}$ & $\nu \mathrm{NN}(36)+\nu \mathrm{NC}(11)$ & $1200 \mathrm{~s}$ & & 1233 & 1205 & 85.54 & 0.69 \\
\hline$v_{44}$ & $\nu \mathrm{NN}(61)+\nu \mathrm{NC}(12)$ & & $1211 \mathrm{~m}$ & 1219 & 1191 & 43.20 & 36.30 \\
\hline$v_{45}$ & $\delta \mathrm{HCC}(71)+\nu \mathrm{CC}(20)$ & & $1170 \mathrm{~m}$ & 1200 & 1173 & 1.88 & 4.90 \\
\hline$v_{46}$ & $\delta \mathrm{HCC}(78)$ & & & 1184 & 1157 & 0.60 & 4.78 \\
\hline$v_{47}$ & $v \mathrm{NC}(41)+\delta \mathrm{HCN}(16)+\delta \mathrm{HCC}(10)$ & & $1126 \mathrm{w}$ & 1164 & 1138 & 5.43 & 2.93 \\
\hline$v_{48}$ & $\delta \mathrm{HCN}(55)$ & $1136 \mathrm{~s}$ & & 1156 & 1130 & 16.14 & 8.01 \\
\hline$\nu_{49}$ & $\delta \mathrm{HCN}(55)+\nu \mathrm{NC}(10)$ & $1105 \mathrm{~m}$ & & 1130 & 1104 & 14.51 & 7.56 \\
\hline$\nu_{50}$ & $\nu \mathrm{CC}(38)+\delta \mathrm{HCC}(28)$ & $1074 \mathrm{w}$ & $1069 \mathrm{w}$ & 1104 & 1079 & 12.00 & 0.64 \\
\hline
\end{tabular}


TABLE 2: Continued.

\begin{tabular}{|c|c|c|c|c|c|c|c|}
\hline \multirow{2}{*}{ Mode } & \multirow{2}{*}{ P.E.D ( $\geq 5 \%)$} & \multicolumn{2}{|c|}{ Experimental } & \multicolumn{4}{|c|}{$\mathrm{B} 3 \mathrm{LYP} / 6-31 \mathrm{G}^{++}(\mathrm{d}, \mathrm{p})$} \\
\hline & & IR & Raman & $\nu^{\alpha}$ & $\nu^{\beta}$ & $I_{\mathrm{IR}}$ & $I_{\mathrm{R}}$ \\
\hline$v_{51}$ & $\nu \mathrm{NC}(35)+\delta \mathrm{HCC}(18)$ & $1060 \mathrm{~m}$ & & 1082 & 1058 & 13.07 & 0.41 \\
\hline$v_{52}$ & $\delta \mathrm{HCC}(83)$ & & & 1071 & 1047 & 0.12 & 2.18 \\
\hline$v_{53}$ & $\delta \mathrm{HCC}(26)+\nu \mathrm{NC}(13)$ & & & 1060 & 1035 & 4.98 & 1.92 \\
\hline$v_{54}$ & $\delta \mathrm{HCC}(59)$ & & & 1057 & 1032 & 0.42 & 0.55 \\
\hline$v_{55}$ & $\nu \mathrm{CC}(45)+\delta \mathrm{CCC}(17)$ & $1026 \mathrm{~m}$ & 1008 vs & 1041 & 1017 & 18.61 & 24.00 \\
\hline$v_{56}$ & $\delta \mathrm{HCC}(80)$ & $1001 \mathrm{w}$ & & 1026 & 1002 & 6.02 & 2.76 \\
\hline$v_{57}$ & $\delta \mathrm{CCC}(62)+\nu \mathrm{CC}(21)$ & & $973 \mathrm{w}$ & 1014 & 990 & 0.17 & 68.48 \\
\hline$v_{58}$ & $\delta \mathrm{HCC}(95)$ & & & 998 & 975 & 0.06 & 0.48 \\
\hline$v_{59}$ & $\delta \mathrm{HCC}(95)$ & & & 980 & 958 & 0.21 & 0.17 \\
\hline$v_{60}$ & $\delta \mathrm{HCC}(32)+\nu \mathrm{CC}(22)+\nu \mathrm{NC}(11)$ & $956 \mathrm{vw}, \mathrm{sh}$ & & 970 & 948 & 13.72 & 0.75 \\
\hline$v_{61}$ & $\nu \mathrm{CC}(87)$ & $932 \mathrm{~s}$ & $946 \mathrm{~m}$ & 954 & 932 & 38.98 & 3.92 \\
\hline$v_{62}$ & $\nu \mathrm{NC}(28)+\delta \mathrm{HCC}(25)$ & & & 943 & 921 & 6.80 & 9.61 \\
\hline$v_{63}$ & $\delta \mathrm{HCC}(79)$ & & & 922 & 901 & 3.20 & 3.83 \\
\hline$v_{64}$ & $\nu \mathrm{CC}(28)+\delta \mathrm{HCC}(27)$ & $892 \mathrm{w}$ & $902 \mathrm{~m}$ & 907 & 886 & 1.89 & 20.36 \\
\hline$v_{65}$ & $\tau \mathrm{HCCN}(95)$ & $842 \mathrm{~m}$ & $858 \mathrm{~m}$ & 848 & 829 & 0.20 & 3.18 \\
\hline$v_{66}$ & $\tau \mathrm{HNCC}(43)$ & $806 \mathrm{w}$ & & 838 & 819 & 6.14 & 4.33 \\
\hline$v_{67}$ & $\tau \mathrm{HCCC}(53)+\gamma \operatorname{CCCC}(16)$ & & $783 w$ & 803 & 785 & 11.75 & 2.48 \\
\hline$v_{68}$ & $\tau \mathrm{HCCC}(73)$ & 763 vs & $749 w$ & 772 & 754 & 42.94 & 1.70 \\
\hline$v_{69}$ & $\gamma \mathrm{OCNC}(81)$ & $744 \mathrm{~m}$ & & 746 & 729 & 13.41 & 4.16 \\
\hline$v_{70}$ & $\nu \mathrm{CC}(35)+\delta \mathrm{CNN}(20)+\tau \mathrm{HCCC}(10)$ & $\begin{array}{l}708 \mathrm{~s} \\
703 \mathrm{~s}\end{array}$ & $705 \mathrm{vw}$ & 728 & 711 & 42.51 & 1.35 \\
\hline$v_{71}$ & $\tau \mathrm{HCCC}(84)$ & & & 701 & 685 & 16.44 & 0.60 \\
\hline$v_{72}$ & $\delta \mathrm{HCC}(25)+\gamma \mathrm{CCCC}(11)$ & & $685 \mathrm{~s}$ & 678 & 662 & 2.43 & 13.74 \\
\hline$v_{73}$ & $\delta \mathrm{HCC}(28)+\delta \mathrm{CNN}(11)$ & $666 \mathrm{~m}$ & $656 \mathrm{~m}$ & 668 & 653 & 6.92 & 5.11 \\
\hline$v_{74}$ & $\delta \operatorname{CCC}(60)$ & $641 \mathrm{~m}$ & & 658 & 643 & 23.86 & 5.80 \\
\hline$v_{75}$ & $\delta \operatorname{CCC}(79)$ & & & 630 & 616 & 1.15 & 7.40 \\
\hline$v_{76}$ & $\tau \operatorname{HNCC}(57)$ & & $603 \mathrm{~s}$ & 1340 & 608 & 56.00 & 27.67 \\
\hline$v_{77}$ & $\gamma \mathrm{CCNN}(21)$ & & & 1325 & 590 & 11.65 & 5.02 \\
\hline$v_{78}$ & $\delta \mathrm{CCN}(30)+\gamma \mathrm{CCNN}(20)+\nu \mathrm{CC}(10)$ & $587 \mathrm{~s}$ & & 1292 & 575 & 25.19 & 15.04 \\
\hline$v_{79}$ & $\delta \mathrm{CCO}(27)+\tau \mathrm{HNCC}(12)+\gamma \mathrm{CCNN}(10)$ & & & 622 & 564 & 5.98 & 4.06 \\
\hline$v_{80}$ & $\gamma \mathrm{NCCC}(76)$ & $501 \mathrm{~s}$ & & 604 & 501 & 8.72 & 1.59 \\
\hline$v_{81}$ & $\delta \mathrm{CCC}(49)$ & & $476 \mathrm{w}$ & 588 & 478 & 8.40 & 2.76 \\
\hline$v_{82}$ & $\tau \operatorname{CCCN}(31)$ & $436 \mathrm{~m}$ & & 577 & 447 & 19.92 & 15.42 \\
\hline$v_{83}$ & $\delta \mathrm{CNC}(26)+\tau \mathrm{CCNC}(23)$ & & $423 \mathrm{~m}$ & 512 & 417 & 1.68 & 4.29 \\
\hline$v_{84}$ & $\tau \mathrm{CCCC}(94)$ & $409 \mathrm{w}$ & $397 \mathrm{vw}$ & 489 & 409 & 0.58 & 1.88 \\
\hline$v_{85}$ & $\delta \mathrm{CCN}(49)$ & $373 \mathrm{~s}$ & & 458 & 383 & 2.79 & 7.76 \\
\hline$v_{86}$ & $\gamma \operatorname{CCCC}(32)$ & & & 427 & 346 & 0.60 & 11.02 \\
\hline$v_{87}$ & $\delta \mathrm{CNN}(57)$ & $331 \mathrm{~m}$ & $335 \mathrm{w}$ & 419 & 336 & 9.39 & 1.00 \\
\hline$v_{88}$ & $\delta \mathrm{CCC}(65)$ & & & 392 & 305 & 1.22 & 3.69 \\
\hline$v_{89}$ & $\delta \mathrm{CCN}(49)$ & $289 \mathrm{~s}$ & $295 \mathrm{w}$ & 354 & 284 & 12.51 & 4.15 \\
\hline$v_{90}$ & $\delta \operatorname{CCC}(35)$ & $261 \mathrm{w}$ & $275 \mathrm{w}$ & 344 & 271 & 1.06 & 1.95 \\
\hline$v_{91}$ & $\tau \mathrm{CCCN}(41)$ & & & 312 & 244 & 1.68 & 3.36 \\
\hline$v_{92}$ & $\delta \mathrm{CCN}(38)+\nu \mathrm{NC}(11)$ & $225 \mathrm{vw}$ & & 290 & 217 & 5.16 & 9.01 \\
\hline$v_{93}$ & $\tau \operatorname{HCCC}(79)$ & & & 277 & 213 & 0.40 & 2.36 \\
\hline$v_{94}$ & $\delta \mathrm{CCN}(37)$ & & $200 \mathrm{~m}$ & 250 & 207 & 3.45 & 21.32 \\
\hline$v_{95}$ & $\tau \mathrm{HCCC}(90)$ & $169 \mathrm{vw}$ & & 222 & 169 & 0.51 & 3.26 \\
\hline$v_{96}$ & $\tau \mathrm{CCCC}(58)$ & & & 218 & 157 & 1.07 & 5.37 \\
\hline$v_{97}$ & $\tau \mathrm{CCNC}(47)$ & $140 \mathrm{~s}$ & & 212 & 141 & 3.32 & 7.37 \\
\hline$v_{98}$ & $\tau \mathrm{HCCC}(54)+\tau \mathrm{CCNC}(11)$ & $134 \mathrm{~s}$ & & 173 & 134 & 0.62 & 23.80 \\
\hline$v_{99}$ & $\tau \mathrm{CCNC}(27)+\tau \mathrm{HCCC}(14)+\delta \mathrm{HCC}(13)$ & $111 \mathrm{~m}$ & & 160 & 113 & 4.87 & 17.07 \\
\hline$\nu_{100}$ & $\tau \mathrm{CNCC}(58)$ & & & 144 & 77 & 1.35 & 138.91 \\
\hline
\end{tabular}


TABLE 2: Continued.

\begin{tabular}{|c|c|c|c|c|c|c|c|}
\hline \multirow{2}{*}{ Mode } & \multirow{2}{*}{ P.E.D ( $\geq 5 \%)$} & \multicolumn{2}{|c|}{ Experimental } & \multicolumn{4}{|c|}{$\mathrm{B} 3 \mathrm{LYP} / 6-31 \mathrm{G}^{++}(\mathrm{d}, \mathrm{p})$} \\
\hline & & IR & Raman & $\nu^{\alpha}$ & $v^{\beta}$ & $I_{\mathrm{IR}}$ & $I_{\mathrm{R}}$ \\
\hline$v_{101}$ & $\tau \mathrm{CNCC}(27)+\tau \mathrm{CCNC}(17)+\delta \mathrm{CCN}(10)$ & $71 \mathrm{vw}$ & & 137 & 68 & 5.61 & 39.27 \\
\hline$v_{102}$ & $\tau \mathrm{CCNN}(80)$ & $60 \mathrm{vw}$ & & 115 & 55 & 1.93 & 72.49 \\
\hline$v_{103}$ & $\tau \mathrm{CNCC}(56)$ & $50 \mathrm{vw}$ & & 79 & 46 & 2.34 & 115.46 \\
\hline$v_{104}$ & $\tau \mathrm{CNCC}(67)$ & $41 \mathrm{vw}$ & & 70 & 39 & 4.55 & 83.80 \\
\hline$\nu_{105}$ & $\tau \mathrm{CCNC}(49)+\delta \mathrm{CNC}(16)$ & & & 57 & 24 & 1.74 & 72.73 \\
\hline
\end{tabular}

$\nu^{\alpha}$ : unscaled wavenumbers, $\nu^{\beta}$ : scaled with 0.955 above $1800 \mathrm{~cm}^{-1}$ and 0.977 under $1800 \mathrm{~cm}^{-1}$. IR and R: calculated infrared and Raman intensities. PED data are taken from VEDA4. v: very, s: strong, m: medium, w: weak, b: broad, and sh: shoulder. $\nu, \delta, \tau$, and $\gamma$ denote stretching, bending, torsion, and out modes, respectively.

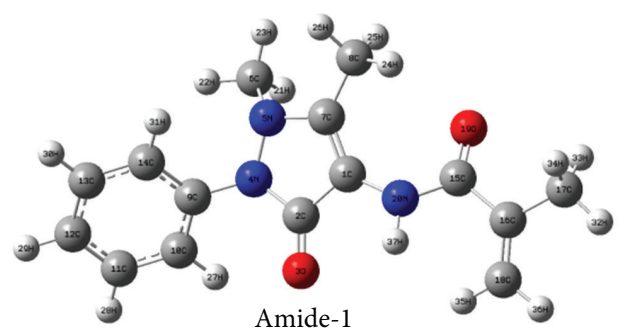

$\Delta G($ Hartree $)=-896.323766$

Relative stability $=0$

Mole fractions $=51 \%$

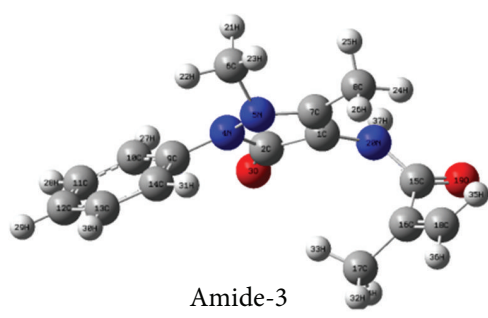

$\Delta G($ Hartree $)=-896.313983$

Relative stability $=6.14$

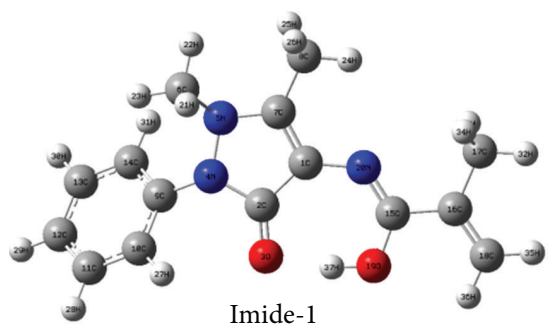

$\Delta G($ Hartree $)=-896.319217$

Relative stability $=2.85$

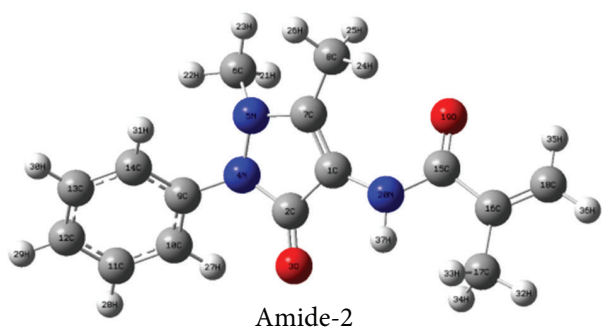

$\Delta G($ Hartree $)=-896.323712$

Relative stability $=0.03$

Mole fractions $=49 \%$

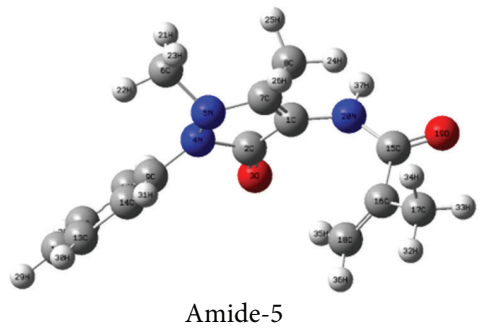

$\Delta G($ Hartree $)=-896.315034$

Relative stability $=5.48$

FIGURE 1: Optimized conformational isomers and numbering of MAAP.

double band, whereas the latter is attributed to the $\mathrm{C}=\mathrm{C}$ band of rings. The corresponding theoretical values of these modes are $1652 \mathrm{~cm}^{-1}$ and $1609 \mathrm{~cm}^{-1}\left(1595 \mathrm{~cm}^{-1}\right)$.

The $\mathrm{CN}$ stretching modes of the amide group are observed at $1491 \mathrm{~cm}^{-1}$ (IR-vs), $1295 \mathrm{~cm}^{-1}$ (IR-vs), $1285 \mathrm{~cm}^{-1}$ (IR-vs), $1245 \mathrm{~cm}^{-1}$ (R-w,sh), and $1060 \mathrm{~cm}^{-1}$ (IR-m) while the present theoretical values are $1520 \mathrm{~cm}^{-1}, 1263 \mathrm{~cm}^{-1}$, and $1058 \mathrm{~cm}^{-1}$. The $\mathrm{CN}$ and $\mathrm{NN}$ stretch vibrations at cyclic ketone position are observed at $1368 \mathrm{~cm}^{-1}$ (IR-s), $1368 \mathrm{~cm}^{-1}$ (Rvs), $1200 \mathrm{~cm}^{-1}$ (IR-s), and $1211 \mathrm{~cm}^{-1}$ (R-m) while the present theoretical values are $1339 \mathrm{~cm}^{-1}, 1205 \mathrm{~cm}^{-1}$, and $1191 \mathrm{~cm}^{-1}$. CC or NC stretching, CCC, CCN, CNC or HCC bending, 
TABLE 3: Complete set of optimized geometric parameters for amide-1 conformational isomer of MAAP.

\begin{tabular}{|c|c|c|c|c|c|c|c|}
\hline \multicolumn{8}{|c|}{$\mathrm{B} 3 \mathrm{LYP} / 6-31 \mathrm{G}^{++}(\mathrm{d}, \mathrm{p})$} \\
\hline Parameters & Amide-1 & Parameters & Amide-1 & Parameters & Amide-1 & Parameters & Amide-1 \\
\hline \multicolumn{2}{|c|}{ Bond lengths $(\AA)$} & \multicolumn{2}{|c|}{ Bond angles $\left({ }^{\circ}\right)$} & \multicolumn{2}{|c|}{ Dihedral angles $\left({ }^{\circ}\right)$} & \multicolumn{2}{|c|}{ Dihedral angles $\left({ }^{\circ}\right)$} \\
\hline $\mathrm{C} 1-\mathrm{C} 2$ & 1.457 & $\mathrm{C} 2-\mathrm{C} 1-\mathrm{C} 7$ & 108.9 & C7-C1-C2-O3 & -173.4 & N4-C9-C10-H27 & 0.6 \\
\hline $\mathrm{C} 1-\mathrm{C} 7$ & 1.362 & $\mathrm{C} 2-\mathrm{C} 1-\mathrm{N} 20$ & 117.8 & $\mathrm{C} 7-\mathrm{C} 1-\mathrm{C} 2-\mathrm{N} 4$ & 3.4 & C14-C9-C10-C11 & -0.2 \\
\hline $\mathrm{C} 1-\mathrm{N} 20$ & 1.400 & C7-C1-N20 & 133.0 & N20-C1-C2-O3 & 1.9 & C14-C9-C10-H27 & -179.2 \\
\hline $\mathrm{C} 2-\mathrm{O} 3$ & 1.232 & $\mathrm{C} 1-\mathrm{C} 2-\mathrm{O} 3$ & 128.2 & N20-C1-C2-N4 & 178.7 & N4-C9-C14-C13 & 179.5 \\
\hline $\mathrm{C} 2-\mathrm{N} 4$ & 1.400 & $\mathrm{C} 1-\mathrm{C} 2-\mathrm{N} 4$ & 105.0 & $\mathrm{C} 2-\mathrm{C} 1-\mathrm{C} 7-\mathrm{N} 5$ & -0.5 & N4-C9-C14-H31 & -1.4 \\
\hline N4-N5 & 1.414 & $\mathrm{O} 3-\mathrm{C} 2-\mathrm{N} 4$ & 126.7 & $\mathrm{C} 2-\mathrm{C} 1-\mathrm{C} 7-\mathrm{C} 8$ & 178.3 & C10-C9-C14-C13 & -0.7 \\
\hline N4-C9 & 1.420 & $\mathrm{C} 2-\mathrm{N} 4-\mathrm{N} 5$ & 109.8 & N20-C1-C7-N5 & -174.9 & C10-C9-C14-H31 & 178.4 \\
\hline N5-C6 & 1.474 & $\mathrm{C} 2-\mathrm{N} 4-\mathrm{C} 9$ & 125.1 & N20-C1-C7-C8 & 4.0 & $\mathrm{C} 9-\mathrm{C} 10-\mathrm{C} 11-\mathrm{C} 12$ & 0.9 \\
\hline N5-C7 & 1.406 & N5-N4-C9 & 119.5 & $\mathrm{C} 2-\mathrm{C} 1-\mathrm{N} 20-\mathrm{C} 15$ & 139.6 & C9-C10-C11-H28 & -179.5 \\
\hline C6-H21 & 1.098 & N4-N5-C6 & 113.1 & C2-C1-N20-H37 & -19.6 & H27-C10-C11-C12 & 179.9 \\
\hline C6-H22 & 1.091 & N4-N5-C7 & 106.7 & C7-C1-N20-C15 & -46.5 & H27-C10-C11-H28 & -0.6 \\
\hline C6-H23 & 1.091 & C6-N5-C7 & 117.3 & C7-C1-N20-H37 & 154.3 & $\mathrm{C} 10-\mathrm{C} 11-\mathrm{C} 12-\mathrm{C} 13$ & -0.7 \\
\hline $\mathrm{C} 7-\mathrm{C} 8$ & 1.494 & N5-C6-H21 & 111.4 & $\mathrm{C} 1-\mathrm{C} 2-\mathrm{N} 4-\mathrm{N} 5$ & -4.9 & $\mathrm{C} 10-\mathrm{C} 11-\mathrm{C} 12-\mathrm{H} 29$ & 179.4 \\
\hline $\mathrm{C} 8-\mathrm{H} 24$ & 1.089 & N5-C6-H22 & 109.1 & $\mathrm{C} 1-\mathrm{C} 2-\mathrm{N} 4-\mathrm{C} 9$ & -158.0 & $\mathrm{H} 28-\mathrm{C} 11-\mathrm{C} 12-\mathrm{C} 13$ & 179.8 \\
\hline $\mathrm{C} 8-\mathrm{H} 25$ & 1.096 & N5-C6-H23 & 108.4 & $\mathrm{O} 3-\mathrm{C} 2-\mathrm{N} 4-\mathrm{N} 5$ & 171.9 & $\mathrm{H} 28-\mathrm{C} 11-\mathrm{C} 12-\mathrm{H} 29$ & -0.1 \\
\hline $\mathrm{C} 8-\mathrm{H} 26$ & 1.095 & H21-C6-H22 & 109.5 & $\mathrm{O} 3-\mathrm{C} 2-\mathrm{N} 4-\mathrm{C} 9$ & 161.2 & C11-C12-C13-C14 & -0.2 \\
\hline C9-C10 & 1.402 & $\mathrm{H} 21-\mathrm{C} 6-\mathrm{H} 23$ & 109.7 & C2-N4-N5-C6 & 135.1 & $\mathrm{C} 11-\mathrm{C} 12-\mathrm{C} 13-\mathrm{H} 30$ & -179.4 \\
\hline C9-C14 & 1.402 & $\mathrm{H} 22-\mathrm{C} 6-\mathrm{H} 23$ & 108.6 & C2-N4-N5-C7 & 4.7 & H29-C12-C13-C14 & 179.6 \\
\hline $\mathrm{C} 10-\mathrm{C} 11$ & 1.395 & $\mathrm{C} 1-\mathrm{C} 7-\mathrm{N} 5$ & 109.3 & C9-N4-N5-C6 & 70.1 & $\mathrm{H} 29-\mathrm{C} 12-\mathrm{C} 13-\mathrm{H} 30$ & 0.4 \\
\hline $\mathrm{C} 10-\mathrm{H} 27$ & 1.083 & $\mathrm{C} 1-\mathrm{C} 7-\mathrm{C} 8$ & 131.4 & C9-N4-N5-C7 & 20.5 & $\mathrm{C} 12-\mathrm{C} 13-\mathrm{C} 14-\mathrm{C} 9$ & 0.9 \\
\hline $\mathrm{C} 11-\mathrm{C} 12$ & 1.398 & $\mathrm{C} 1-\mathrm{C} 7-\mathrm{N} 5$ & 109.3 & $\mathrm{C} 2-\mathrm{N} 4-\mathrm{C} 9-\mathrm{C} 10$ & -50.7 & $\mathrm{C} 12-\mathrm{C} 13-\mathrm{C} 14-\mathrm{H} 31$ & -178.2 \\
\hline $\mathrm{C} 11-\mathrm{H} 28$ & 1.086 & N5-C7-C8 & 119.2 & C2-N4-C9-C14 & 129.1 & H30-C13-C14-C9 & -179.9 \\
\hline $\mathrm{C} 12-\mathrm{C} 13$ & 1.397 & C7-C8-H24 & 109.9 & N5-N4-C9-C10 & 158.6 & H30-C13-C14-H31 & 1.0 \\
\hline $\mathrm{C} 12-\mathrm{H} 29$ & 1.086 & C7-C8-H25 & 111.7 & N5-N4-C9-C14 & -21.6 & O19-C15-C16-C17 & 31.4 \\
\hline $\mathrm{C} 13-\mathrm{C} 14$ & 1.396 & C7-C8-H26 & 110.4 & N4-N5-C6-H21 & -62.2 & O19-C15-C16-C18 & -144.2 \\
\hline C13-H30 & 1.086 & $\mathrm{H} 24-\mathrm{C} 8-\mathrm{H} 25$ & 106.6 & N4-N5-C6-H22 & 58.8 & N20-C15-C16-C17 & -147.4 \\
\hline C14-H31 & 1.084 & $\mathrm{H} 24-\mathrm{C} 8-\mathrm{H} 26$ & 109.3 & N4-N5-C6-H23 & 176.9 & N20-C15-C16-C18 & 37.0 \\
\hline $\mathrm{C} 15-\mathrm{C} 16$ & 1.508 & $\mathrm{H} 25-\mathrm{C} 8-\mathrm{H} 26$ & 108.9 & C7-N5-C6-H21 & 62.6 & $\mathrm{C} 16-\mathrm{C} 15-\mathrm{N} 20-\mathrm{C} 1$ & -165.9 \\
\hline C15-O19 & 1.229 & $\mathrm{~N} 4-\mathrm{C} 9-\mathrm{C} 10$ & 118.9 & C7-N5-C6-H22 & -176.3 & C16-C15-N20-H37 & -7.4 \\
\hline $\mathrm{C} 15-\mathrm{N} 20$ & 1.377 & N4-C9-C14 & 120.9 & C7-N5-C6-H23 & -58.2 & O19-C15-N20-C1 & 15.4 \\
\hline $\mathrm{C} 16-\mathrm{C} 17$ & 1.506 & C10-C9-C14 & 120.2 & N4-N5-C7-C1 & -2.5 & O19-C15-N20-H37 & 173.8 \\
\hline $\mathrm{C} 16-\mathrm{C} 18$ & 1.342 & C9-C10-C11 & 119.5 & N4-N5-C7-C8 & 1.6 & C15-C16-C17-H32 & -175.8 \\
\hline C17-H32 & 1.093 & $\mathrm{C} 9-\mathrm{C} 10-\mathrm{H} 27$ & 119.6 & C6-N5-C7-C1 & -130.5 & C15-C16-C17-H33 & -54.7 \\
\hline C17-H33 & 1.094 & $\mathrm{C} 11-\mathrm{C} 10-\mathrm{H} 27$ & 120.9 & C6-N5-C7-C8 & 129.5 & C15-C16-C17-H34 & 63.2 \\
\hline C17-H34 & 1.097 & $\mathrm{C} 10-\mathrm{C} 11-\mathrm{C} 12$ & 120.7 & C1-C7-C8-H24 & -21.7 & C18-C16-C17-H32 & -0.3 \\
\hline C18-H35 & 1.086 & $\mathrm{C} 10-\mathrm{C} 11-\mathrm{H} 28$ & 119.2 & C1-C7-C8-H25 & 96.4 & C18-C16-C17-H33 & 120.9 \\
\hline C18-H36 & 1.086 & $\mathrm{C} 12-\mathrm{C} 11-\mathrm{H} 28$ & 120.1 & C1-C7-C8-H26 & -142.3 & C18-C16-C17-H34 & -121.2 \\
\hline \multirow[t]{10}{*}{ N20-H37 } & 1.014 & C11-C12-C13 & 119.5 & N5-C7-C8-H24 & 157.1 & C15-C16-C18-H35 & -1.0 \\
\hline & & $\mathrm{C} 11-\mathrm{C} 12-\mathrm{H} 29$ & 120.3 & N5-C7-C8-H25 & -84.8 & C15-C16-C18-H36 & 176.8 \\
\hline & & $\mathrm{C} 13-\mathrm{C} 12-\mathrm{H} 29$ & 120.3 & N5-C7-C8-H26 & 36.5 & C17-C16-C18-H35 & -176.2 \\
\hline & & $\mathrm{C} 12-\mathrm{C} 13-\mathrm{C} 14$ & 120.5 & N4-C9-C10-C11 & 179.6 & $\mathrm{C} 17-\mathrm{C} 16-\mathrm{C} 18-\mathrm{H} 36$ & 1.5 \\
\hline & & $\mathrm{C} 12-\mathrm{C} 13-\mathrm{H} 30$ & 120.2 & & & & \\
\hline & & $\mathrm{C} 14-\mathrm{C} 13-\mathrm{H} 30$ & 119.3 & & & & \\
\hline & & C9-C14-C13 & 119.6 & & & & \\
\hline & & C9-C14-H31 & 119.7 & & & & \\
\hline & & $\mathrm{C} 13-\mathrm{C} 14-\mathrm{H} 31$ & 120.7 & & & & \\
\hline & & C16-C15-O19 & 121.3 & & & & \\
\hline
\end{tabular}


TABle 3: Continued.

\begin{tabular}{|c|c|c|c|c|c|c|}
\hline \multicolumn{7}{|c|}{ B3LYP/6-31G ${ }^{++}(\mathrm{d}, \mathrm{p})$} \\
\hline Parameters & Parameters & Amide-1 & Parameters & Amide-1 & Parameters & Amide-1 \\
\hline Bond lengths $(\AA)$ & \multicolumn{2}{|c|}{ Bond angles $\left({ }^{\circ}\right)$} & Dihedra & les $\left({ }^{\circ}\right)$ & \multicolumn{2}{|c|}{ Dihedral angles $\left({ }^{\circ}\right)$} \\
\hline & $\mathrm{C} 16-\mathrm{C} 15-\mathrm{N} 20$ & 115.9 & & & & \\
\hline & O19-C15-N20 & 122.8 & & & & \\
\hline & C15-C16-C17 & 114.8 & & & & \\
\hline & C15-C16-C18 & 121.7 & & & & \\
\hline & C17-C16-C18 & 123.4 & & & & \\
\hline & C16-C17-H32 & 111.0 & & & & \\
\hline & C16-C17-H33 & 110.1 & & & & \\
\hline & C16-C17-H34 & 111.0 & & & & \\
\hline & H32-C17-H33 & 109.3 & & & & \\
\hline & H32-C17-H34 & 108.6 & & & & \\
\hline & H33-C17-H34 & 106.7 & & & & \\
\hline & $\mathrm{C} 16-\mathrm{C} 18-\mathrm{H} 35$ & 122.9 & & & & \\
\hline & C16-C18-H36 & 120.8 & & & & \\
\hline & $\mathrm{H} 35-\mathrm{C} 18-\mathrm{H} 36$ & 116.2 & & & & \\
\hline & C1-N20-C15 & 126.2 & & & & \\
\hline & C1-N20-H37 & 113.2 & & & & \\
\hline & C15-N20-H37 & 117.5 & & & & \\
\hline
\end{tabular}

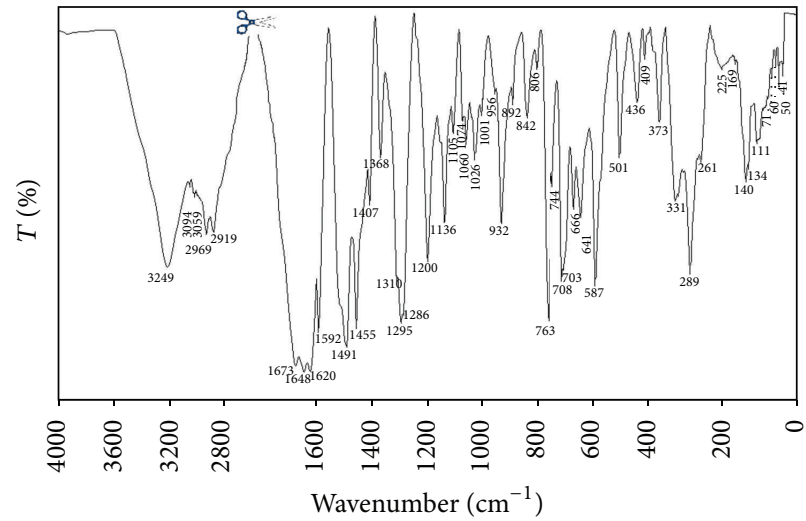

(a)

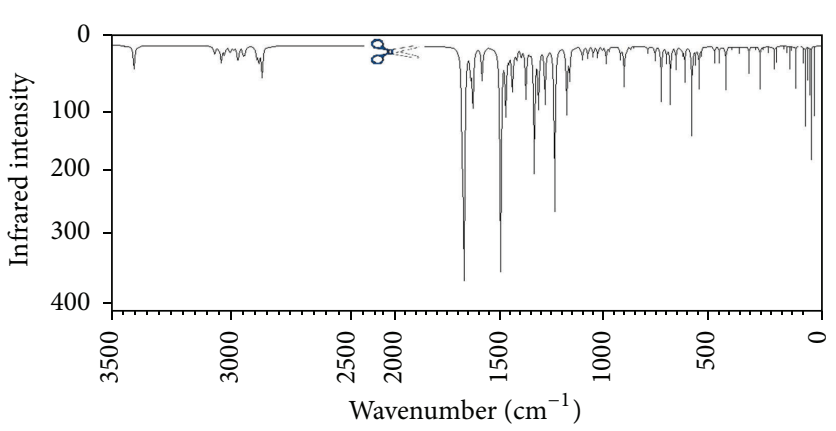

(c)

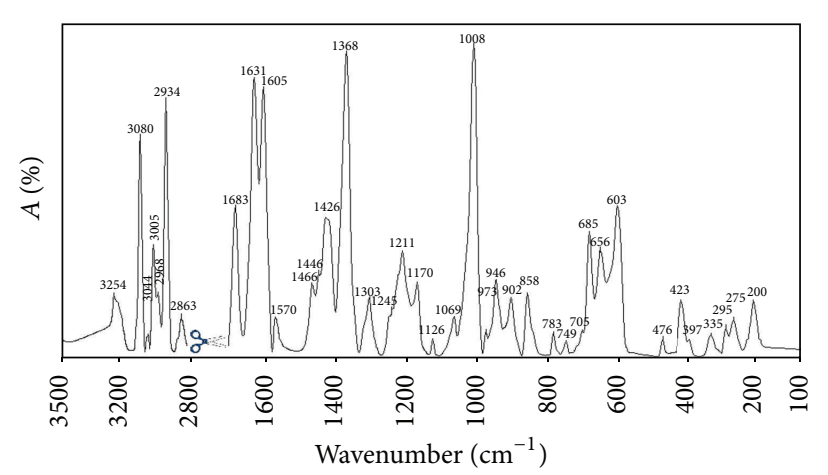

(b)

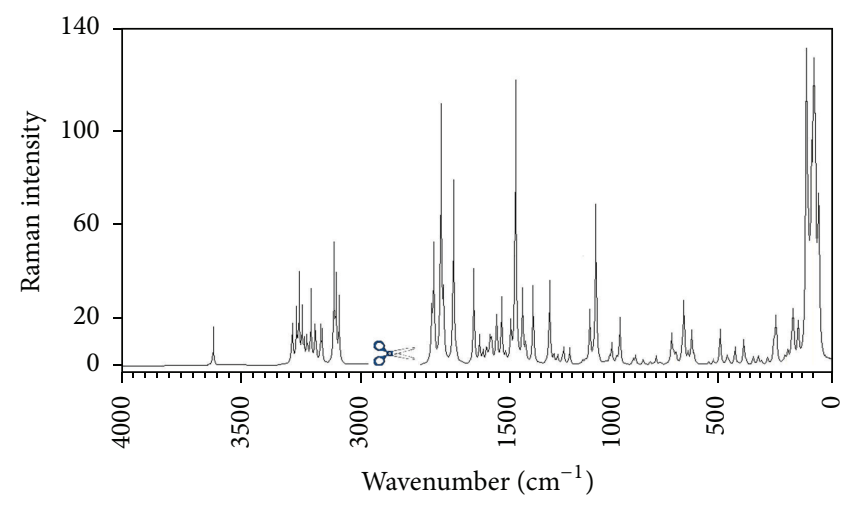

(d)

Figure 2: Experimental ((a), (b)) and theoretical ((c), (d)) vibrational spectra of MAAP. 
some torsion, and out modes dominate the regions of 1000$500 \mathrm{~cm}^{-1}$ while $\mathrm{CCC}, \mathrm{CCN}, \mathrm{CNC}$, or $\mathrm{CNN}$ bending and $\mathrm{CCCN}, \mathrm{CCNC}$, CNCC, CCNN, HCCC, or CCCC torsion modes are seen in the low frequency region. Similar situations have been shown in for calculations. Vibrational modes in the low wavenumber region of the spectrum contain contributions of several internal coordinates and their assignments have reduction approximation to one of two of the internal coordinates.

To make a comparison between the experimental and theoretical frequencies, we have calculated RMSD. This is used to measure the difference between values predicted by a model and those actually observed from the thing being modeled. In this study, RMSD values have been obtained as $29 \mathrm{~cm}^{-1}$ (IR) and $32 \mathrm{~cm}^{-1}$ (R). Omitting the $v_{1}$ stretch from the analysis results substantially reduced RMSD values as $15 \mathrm{~cm}^{-1}$ (IR) and $17 \mathrm{~cm}^{-1}$ (R). Furthermore, the correlation values between the experimental and calculated vibrational frequencies are found to be as 0.99898 (IR) and 0.99889 (R). Similarly, omitting the $v_{1}$ stretch from the analysis results substantially reduced correlation values as 0.99963 (IR) and 0.99960 (R). It can be seen that the B3LYP calculation is reliable for both IR and Raman spectra.

Regarding the calculated fundamentals, in general, the computed vibrational intensities are in agreement with the experimental results. Although the calculated vibrational intensities of some modes are about zero, these bands can be seen in the vibrational spectra. The opposite situation has also been observed. Similarly, the $v_{42}$ and $v_{70}$ modes are observed as double peaks in the infrared spectrum which can be attributed to molecular interactions (Figure 2). It is also important to note that theoretical studies were conducted for an isolated molecule in the gaseous state contrary to the experimental values recorded for the presence of interactions in the solid phase.

The HOMO and LUMO orbitals are called the frontier orbitals and determine the way the molecule interacts with other species. The HOMO is the orbital that could behave as an electron donor, since it is the outermost orbital containing electrons. The LUMO is the orbital that could act as the electron acceptor, as it is the innermost orbital that has room to accept electrons. The transitions can be described from HOMO to LUMO. A single orbital, however, may be both the LUMO and the HOMO. The HOMO is dominated by nitrogen, oxygen, and carbon atoms. The LUMO is located over all atoms except from some $\mathrm{CH}_{3}$ and $\mathrm{NH}$ groups in MAAP. Frontier molecular orbital and their orbital energy are shown in Figure 3 together with the HOMO-LUMO gap. The energy gap given from the ground state to first excited state is calculated at around $4.66 \mathrm{eV}$. The laser used for Raman analysis in the present study has an energy around half of this gap. Therefore, electronic excitement due to Raman laser seems unlikely.

\section{Conclusion}

The experimental and theoretical vibrational investigations of MAAP are performed, for the first time, by using FT-IR,

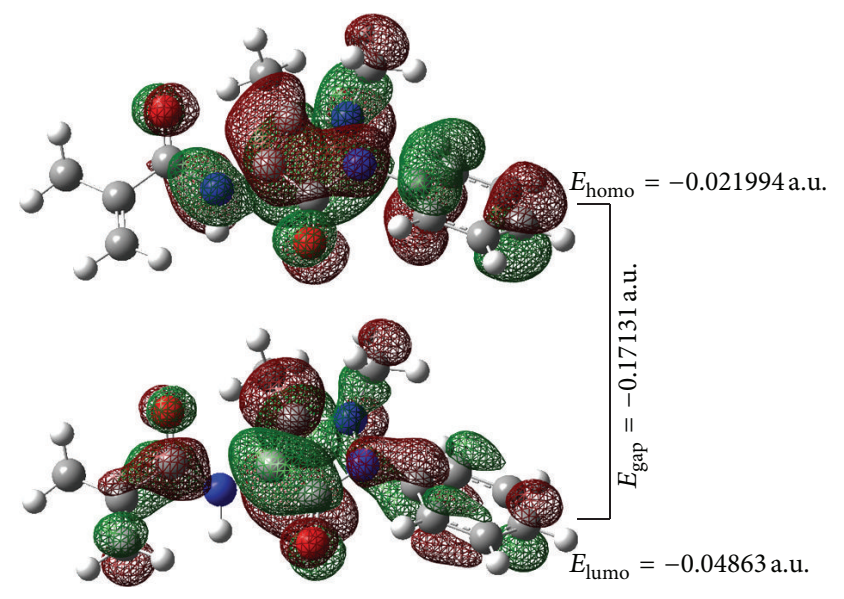

FIgURE 3: Frontier molecular orbitals for MAAP.

Raman, and quantum chemical calculations. In conclusion, the following results can be summarized.

(1) Results of energy calculations for gas phase indicate that the amide- 1 conformational isomer is the most stable conformer of MAAP. Furthermore, relative energies of the other five conformational isomers, except for the amide-2, are larger than $2.0 \mathrm{kcal} / \mathrm{mol}$. Therefore, relative mole fractions of the five forms could be neglected, and these results suggest that the MAAP molecule prefers the amide- 1 and amide2 conformational isomers with preference of $51 \%$ and $49 \%$, respectively, and the conformational energy barrier is independent of the imide form.

(2) The RMSD and correlation values between the experimental and calculated vibrational frequencies indicate that $\mathrm{B} 3 \mathrm{LYP} / 6-31 \mathrm{G}^{++}(\mathrm{d}, \mathrm{p})$ method is reliable, and this theoretical approximation makes the understanding of vibrational spectrum of MAAP easier.

\section{Conflict of Interests}

The authors declare that no conflict of interests exists.

\section{References}

[1] A. Ersöz, A. Denizli, İ. Şener, A. Atılır, S. Diltemiz, and R. Say, "Removal of phenolic compounds with nitrophenol-imprinted polymer based on $\phi-\phi$ and hydrogen-bonding interactions," Separation and Purification Technology, vol. 38, no. 2, pp. 173179, 2004.

[2] R. Say, "Creation of recognition sites for organophosphate esters based on charge transfer and ligand exchange imprinting methods," Analytica Chimica Acta, vol. 579, no. 1, pp. 74-80, 2006.

[3] Z. X. Xu, H. J. Gao, L. M. Zhang, X. Q. Chen, and X. G. Qiao, "The biomimetic immunoassay based on molecularly imprinted polymer: a comprehensive review of recent progress and future prospects," Journal of Food Science, vol. 76, no. 2, pp. R69-R75, 2011. 
[4] A. Ersöz, S. E. Diltemiz, A. A. Özcan, A. Denizli, and R. Say, "8-OHdG sensing with MIP based solid phase extraction and QCM technique," Sensors and Actuators B, vol. 137, no. 1, pp. 711, 2009.

[5] R. Say, A. Gültekin, A. A. Özcan, A. Denizli, and A. Ersöz, "Preparation of new molecularly imprinted quartz crystal microbalance hybride sensor system for 8-hydroxy-2' deoxyguanosine determination," Analytica Chimica Acta, vol. 640, no. 1-2, pp. 82-86, 2009.

[6] S. H. Lin and R. S. Juang, "Adsorption of phenol and its derivatives from water using synthetic resins and low-cost natural adsorbents: a review," Journal of Environmental Management, vol. 90, no. 3, pp. 1336-1349, 2009.

[7] A. P. Scott and L. Radom, "Harmonic vibrational frequencies: an evaluation of Hartree-Fock, Møller-Plesset, Quadratic Configuration Interaction, Density Functional Theory, and Semiempirical Scale Factors," The Journal of Physical Chemistry, vol. 100, no. 41, pp. 16502-16513, 1996.

[8] G. Rauhut and P. Pulay, "Transferable scaling factors for density functional derived vibrational force fields," Journal of Physical Chemistry, vol. 99, no. 10, pp. 3093-3100, 1995.

[9] J. R. Durig, A. Ganguly, A. M. El Defrawy et al., "Conformational stability, r0 structural parameters, barriers to internal rotation and vibrational assignment of cyclobutylamine," Journal of Molecular Structure, vol. 918, no. 1-3, pp. 64-76, 2009.

[10] E. Güneş and C. Parlak, "DFT, FT-Raman and FT-IR investigations of 5-methoxysalicylic acid," Spectrochimica Acta A, vol. 82, no. 1, pp. 504-512, 2011.

[11] C. Parlak, "Theoretical and experimental vibrational spectroscopic study of 4-(1-Pyrrolidinyl)piperidine," Journal of Molecular Structure, vol. 966, no. 1-3, pp. 1-7, 2010.

[12] Ö. Alver and C. Parlak, "Vibrational spectroscopic investigation and conformational analysis of 1-pentylamine: A Comparative Density Functional Study," Journal of Theoretical and Computational Chemistry, vol. 9, no. 3, pp. 667-685, 2010.

[13] Ö. Alver and C. Parlak, "DFT, FT-Raman, FT-IR, liquid and solid state NMR studies of 2,6-dimethoxyphenylboronic acid," Vibrational Spectroscopy, vol. 54, no. 1, pp. 1-9, 2010.

[14] Ö. Bağlayan, M. F. Kaya, C. Parlak, and M. Şenyel, "FT-IR and Raman spectroscopic and quantum chemical investigations of some metal halide complexes of 1-phenylpiperazine," Spectrochimica Acta A, vol. 88, pp. 144-155, 2012.

[15] M. J. Frisch, G. W. Trucks, H. B. Schlegel et al., Gaussian 09, Revision A. 1, Gaussian, Wallingford, Conn, USA, 2009.

[16] R. D. Dennington, T. A. Keith, and J. M. Millam, GaussView 5. 0. 8, Gaussian, 2008.

[17] Spartan 10, Version 1.10, 92612, Wavefunction, Irvine, Calif, USA, 2011.

[18] M. H. Jamróz, "Vibrational energy distribution analysis: VEDA 4 program," Warsaw, 2004, http://smmg.pl/index.php/software/ sowtware-veda.html.

[19] T. Hökelek, Z. Kılıç, M. Işıklanand, and M. Hayvalı, "Crystal Structure of 4-[(1E)-(2-Hydroxynaphthyl)methylidene]amino1,5-dimethyl-2-phenyl-2,3-dihydro- $1 H$-pyrazol-3-one," Analytical Science, vol. 18, no. 2, pp. 215-216, 2002.

[20] M. J. Percinoa, V. M. Chapela, C. Rodriguez-Barbarin, and S. Bernes, "X-ray crystal structure of two different phases (triclinic and orthorhombic) of $p$-methacryloylaminophenylarsonic acid monomer," Journal of Molecular Structure, vol. 562, no. 1-3, pp. 45-53, 2001.
[21] N. A. Besley, "Ab initio modeling of amide vibrational bands in solution," Journal of Physical Chemistry A, vol. 108, no. 49, pp. 10794-10800, 2004. 

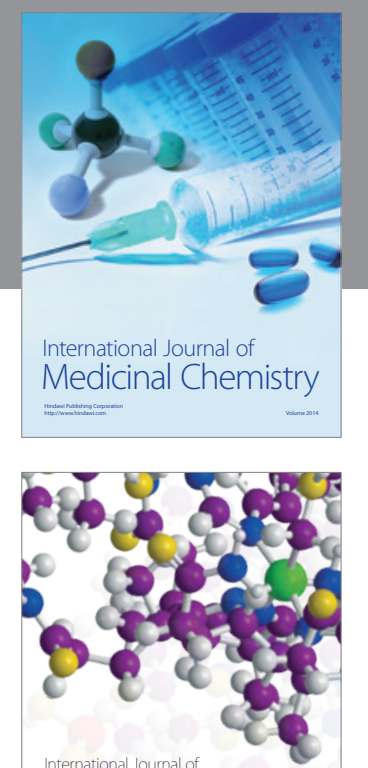

\section{Carbohydrate} Chemistry

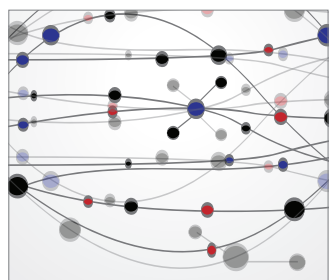

The Scientific World Journal
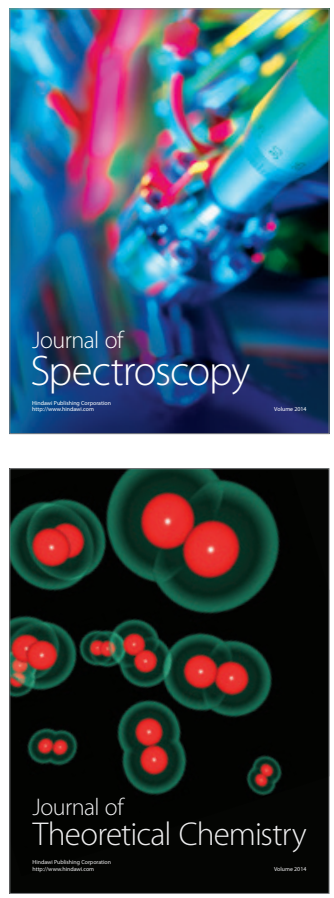
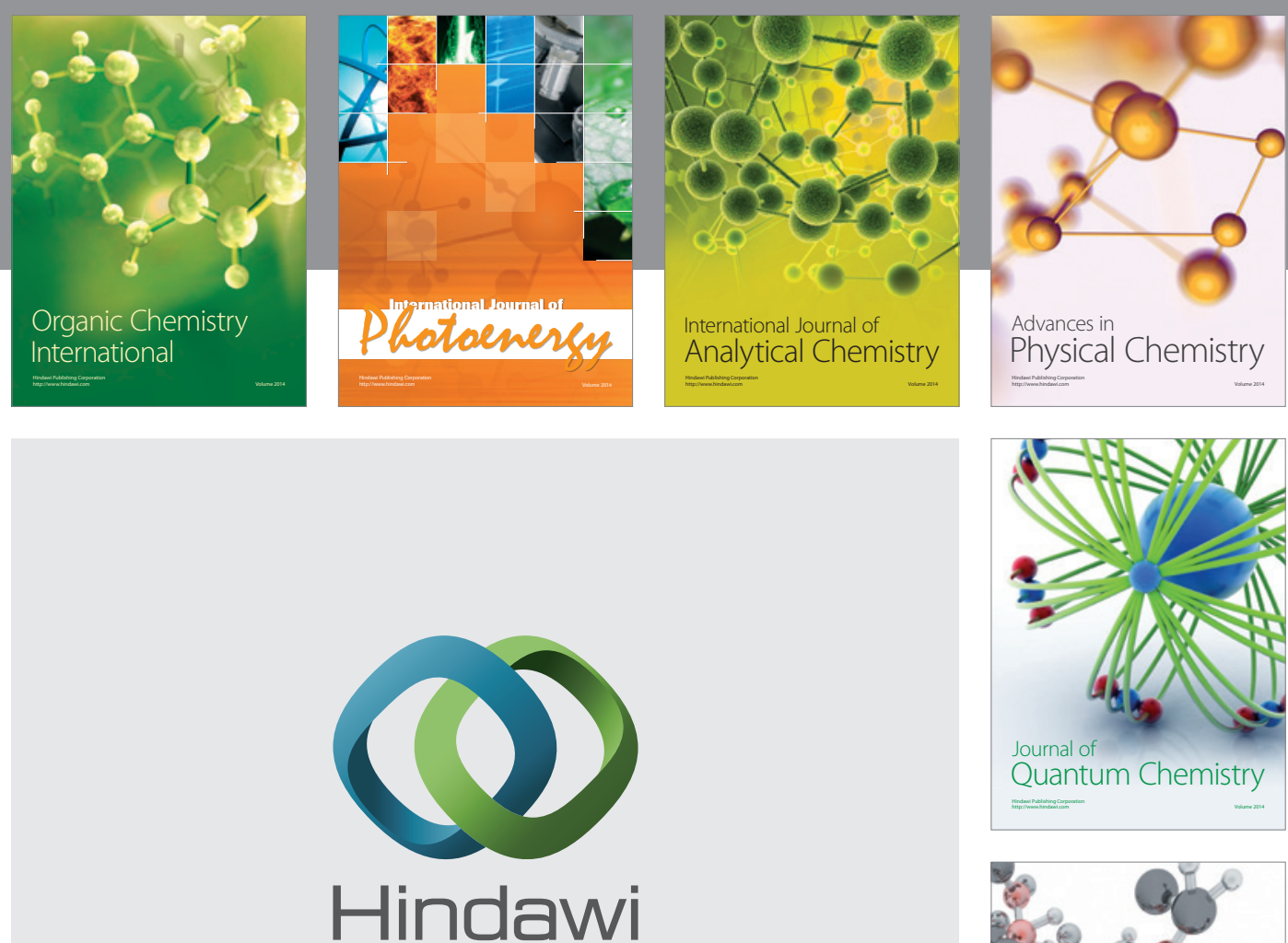

Submit your manuscripts at

http://www.hindawi.com

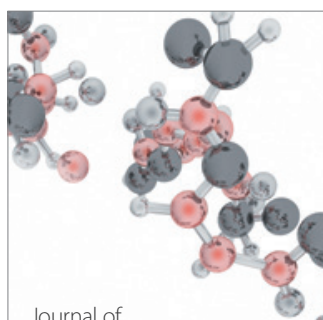

Analytical Methods

in Chemistry

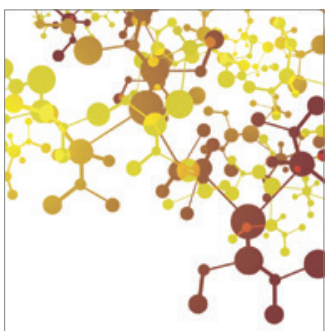

Journal of

Applied Chemistry

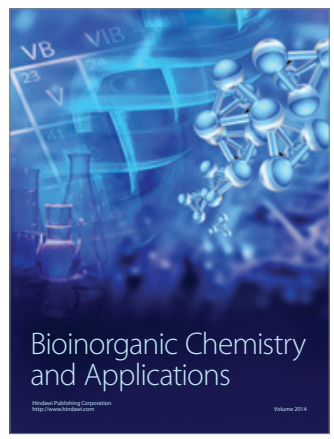

Inorganic Chemistry
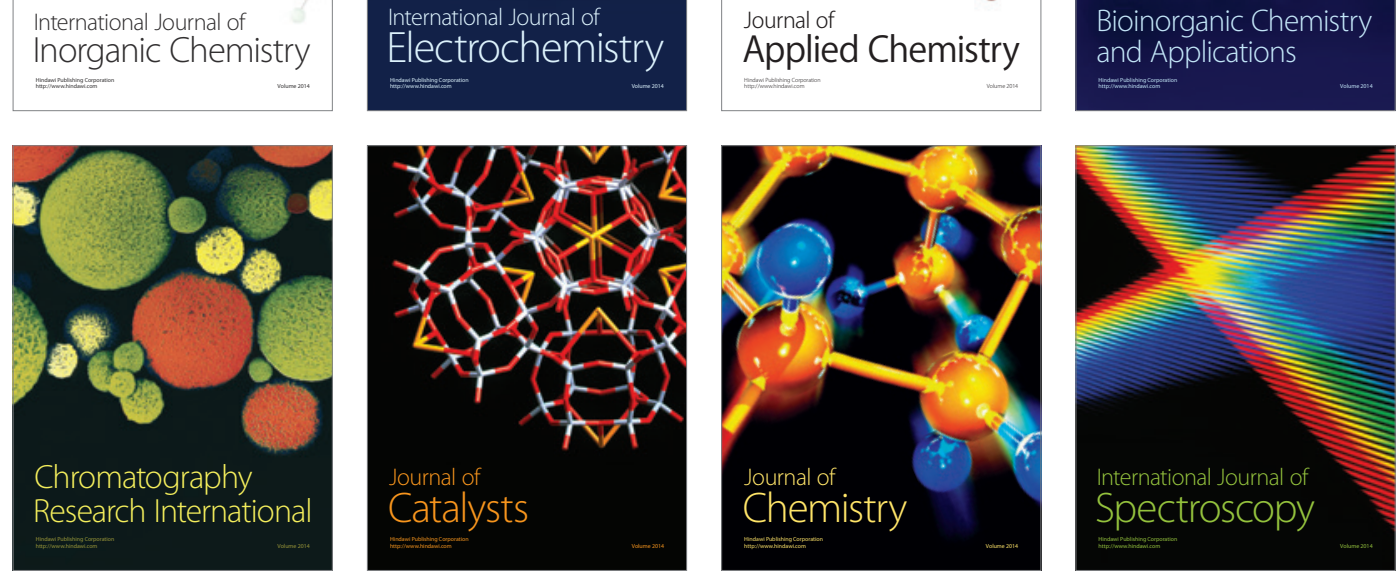